\title{
Nephroprotective Effects of L-Arginine against Chemotherapy Induced Acute Kidney Injury in Wistar Rats
}

\author{
Kumayl Abbas Meghji, ${ }^{1}$ Tariq Feroz Memon, ${ }^{2}$ Imtiaz Ahmed, ${ }^{3}$ Sehar Gul Memon, ${ }^{4}$ Naila Noor, ${ }^{5}$ Ali Abbas ${ }^{6}$ \\ ${ }^{1}$ Assistant Professor, Department of Physiology, Isra University, Hyderabad Pakistan \\ ${ }^{2}$ Assistant Professor, Department of Community Medicine, Isra University, Hyderabad Pakistan \\ ${ }^{3}$ Assistant Professor, Department of Biochemistry, Suleman Roshan Medical College, Tando Ādam, Pakistan \\ ${ }^{4}$ Lecturer, Department of Physiology, Ghad College, Riyadh, Saudi Arabia \\ ${ }^{5}$ Senior Lecturer, Department of Physiology, Isra University, Hyderabad Pakistan \\ ${ }^{6}$ Assistant Professor, Department of Pharmacology, Isra University, Hyderabad Pakistan
}

\begin{abstract}
A B ST RACT
Background: L-arginine is a nitric oxide precursor that decreases oxidative stress and regulates the blood flow by modifying the tone of vessels and also prevent ischemic renal tissue injuries. The objective of this study was to evaluate the protective role of L-arginine in cisplatin-induced acute renal injury through assessment of renal, oxidative stress, and inflammatory markers in albino Wistar rats.

Material and Methods: A quasi-experimental study was conducted at the Department of Physiology and Postgraduate Research Laboratory, Isra University, Hyderabad, Pakistan from April to September 2019. Thirty male Albino Wistar rats were selected through non-random purposive sampling and divided equally into three different groups: Group $A$ (control group), Group B (cisplatin group), and Group C (cisplatin plus arginine group). Blood samples from rats were collected through cardiac puncture, followed by renal histopathological analysis under light microscope. The severity of tissue damage was observed using a graded scale from ' 0 ' (no damage) to III (severe damage). Data was analyzed using SPSS version 24.0., with one-way ANOVA and Post hoc Tukey analysis used for comparison of different variables.

Results: There was a statistically significant decline in the bodyweight and rise in absolute kidney weight of group $B$ in comparison with the other two groups $(P<.05)$. Moreover, a significant rise in serum renal markers was observed in group $\mathrm{B}(P<.05)$, with a statistically significant decline in these markers in group $C$ compared with group $B(P<.05)$. Marked histological alterations (glomerular and tubular damage) were observed in renal tissues of group $B$ as compared to group C.

Conclusions: The present study concludes that L-arginine exerts anti-oxidative, anti-inflammatory, and nephroprotective effects against renal tissue damage caused by Cisplatin.

Key words: Acute kidney injury, Antioxidant, Cisplatin, L-Arginine, Oxidative stress

$\begin{array}{lll}\text { Authors' Contribution: } & \text { Correspondence: } \\ { }^{1} \text { Conception; Literature research; } & \text { Tariq Feroz Memon } \\ \text { manuscript design and drafting; }{ }^{2-6} \text { Critical } & \text { Email: drtariqferoz@gmail.com } \\ \text { analysis and manuscript review; Data } & \end{array}$

analysis and manuscript review; Data

analysis; Manuscript Editing.

Article info:

Received: June 19, 2020

Accepted: December 7, 2020
\end{abstract}

Cite this article. Maghji KA, Memon TF, Ahmed I, Memon SG, Noor $N$, Abbas A. Nephroprotective Effects of L-Arginine against Chemotherapy Induced Acute Kidney Injury in Wistar Rats. J Islamabad Med Dental Coll. 2020; 9(4): 249-255. Doi: 10.35787/jimdc.v9i4.535
Funding Source: Nil

Conflict of Interest: Nil 


\section{Introduction}

Kidney disorders are recognized as a major public health concern as the number of such diseases is significantly increasing each year. According to reports, the prevalence of diseases related to kidneys has climbed up by $10 \%$ in the general population and up to $50 \%$ in high-risk subpopulations in the last 10 years. ${ }^{1}$ Acute kidney injury (AKI), previously known as acute renal failure is characterized by a sudden decline in renal function resulting in the accumulation of metabolic wastes such as urea and creatinine in the blood. ${ }^{2}$ Although the etiology of AKI is diverse (e.g., sepsis, ischemia/hypo-perfusion, etc.), nephrotoxic drugs including chemotherapeutic agents like Cisplatin, are the main causative factors, as demonstrated in $19 \%$ to $25 \%$ of cases. $^{3}$

Cisplatin is a very common and effective antineoplastic drug used against a wide range of cancers. Along with its effective use, it also poses many harmful effects on the tissues due to its reactive oxygen species (ROS) generating capability that causes depletion of glutathione levels and inhibition of renal antioxidant enzymes resulting in nephrotoxicity. Studies reported nephrotoxicity in $25 \%$ to $35 \%$ of patients who received a single dose of Cisplatin. ${ }^{4,5}$

The pathogenesis of AKI has been widely attributed to defects in the L-Arginine: Nitric oxide (NO) pathway. ${ }^{6}$ L-Arginine, a NO precursor, is converted into NO by the action of the enzyme NO synthase (NOS). ${ }^{7}$ NO decreases oxidative stress as well as regulates blood flow by modifying the vascular tone. ${ }^{8}$ It prevents the ischemic tissue injuries of different organs like kidneys, heart, lungs, liver, and intestine of experimental animals. It also decreases neutrophil and leukocyte adhesion as well as decreases the secretion of pro-inflammatory cytokines, such as C-reactive protein (CRP), which play an important role in causing tissue injury. ${ }^{8,9}$

The present study was designed with an objective to evaluate the protective role of L-arginine in cisplatininduced acute renal injury through assessment of renal, oxidative stress, and inflammatory markers in albino Wistar rats.

\section{Material and Methods}

This quasi-experimental study was carried out in the Department of Physiology and Postgraduate Research Laboratory, Isra University, Hyderabad, Pakistan from April to September 2019. About thirty healthy, male Albino Wistar rats, aged 8-10 weeks and weighing between 250 to 300 grams, were procured from the Agriculture University of Tando Jam, Sindh by non-random purposive sampling technique. The sample size was calculated using the standard method of power analysis for animal studies. ${ }^{10,11}$ This study was approved by the Ethics Review Committee of Isra University, that is fully equipped to give permission for animal studies. All the animals were handled according to the guidelines of International Research Council for laboratory animals' handling.

Before the initiation of the experiment, animals were kept for ten days of acclimatization in a wellequipped and hygienic environment at the postgraduate laboratory in Isra University, Hyderabad at the optimum temperature of $24-26^{\circ} \mathrm{C}$ in a day-night cycle of $12 / 12$ hours. To avoid any harm, animals were placed in plastic cages with water drinkers having stainless steel nozzles and feed containers. Rats were provided free access to chow diet and clean water ad libitum. Their bedding consisted of sawdust and was renewed daily. 
All rats were equally $(n=10)$ divided into Group A (control group, given a normal chow diet and clean water ad libitum), Group B (single dose of Cisplatin, $20 \mathrm{mg} / \mathrm{kg}$ body weight, intraperitoneal), and Group $\mathrm{C}$ (single dose of cisplatin, 20mg/kg + L-Arginine, 10 $\mathrm{mg} / \mathrm{kg}$, body weight, intraperitoneal). Arginine was crushed into powder and administered orally, mixed in diet, for 3 weeks. The level of the orally administered dosage of arginine $(10 \mathrm{mg} / \mathrm{kg})$ and the intraperitoneal dose of Cisplatin (Cisplatin $20 \mathrm{mg} / \mathrm{kg}$ ) was based on previous studies. ${ }^{12,13}$ Soon after the acclimatization period, the bodyweight of all rats was measured twice i.e. before initiation of the experiment and after completion of three weeks of the experiment using an electronic precision balance. On completion of the experiment, all rats were given anesthesia (Inj. Sodium pentobarbital at $40 \mathrm{mg} / \mathrm{kg}$, intraperitoneal) and sacrificed by cervical dislocation. For analysis of oxidative and renal function markers, blood was collected by cardiac puncture.

Serum urea and creatinine were analyzed on Hitachi Roche automated chemistry analyzer (Cobas c311) whereas serum glutathione peroxidase (GPX) was analyzed using Bioassay Technology ELISA kit (catalog no: E1242Ra) with the results read through Dia Source ELISA plate reader (RT0931117DIA) at Diagnostic Laboratory, Isra University, Hyderabad.

The kidneys were dissected out and weighed on an electronic scale after which they were fixed in $10 \%$ buffered formalin. Then, they were passed in xylene for clearing and embedded in paraffin wax. Thin tissue sections, of up to 4- $\mu \mathrm{m}$ thickness were cut manually using rotary microtome, 290 and stained with Hematoxylin and Eosin (H\&E) for examination under the light microscope (Olympus BX51, Tokyo, Japan). Histopathological analysis of renal tissue was done by evaluating the degree of infiltration of inflammatory cells, vascular congestion, hydropic change (swelling of epithelial cells), etc. The changes in severity of tissue damage were observed using a graded scale adopted from a previous study as: none (0), mild (I), moderate (II), and severe (III). ${ }^{14}$

Statistical analysis of data was performed in SPSS version 24.0. Findings of variables like body and kidney weights, oxidative, inflammatory, and renal function markers were expressed as mean and standard deviation while their comparison was analyzed by one-way ANOVA and Post hoc Tukey analysis. The level of significance was set at $P$-value $\leq .05$.

\section{Results}

Table I shows changes in the pre- and postexperimental body weights and absolute kidney weights of albino Wistar rats. There was a statistically significant decline in the bodyweight of group $B$ in comparison with groups $A$ and $C(P<.05)$. Similarly, a significant rise in absolute kidney weight was observed in group $B$ in comparison with groups $A$ and $C(P<.001)$. However, the difference between absolute kidney weights of groups $A$ and $C$ was nonsignificant (Table I).

The changes in markers of renal function, oxidative stress as well as inflammation are shown in Table II. There was a significant rise in serum urea, creatinine, and CRP levels in group B in comparison with groups A and C. However, the experimental animals which received L-arginine only revealed markedly decreased blood urea, creatinine, and CRP levels as compared to group B. Similarly, there was a statistically significant decline in the serum Glutathione Peroxidase (GPX) levels of group $B$ in comparison with groups A and C (Table II).

Figures 1, 2, and 3 demonstrate the photomicrographic findings of cut-sections of renal tissues of groups A, B, and C, respectively. Prominent glomerular structure shrinkage, dilatation of the Bowman's capsule, peritubular fibrotic areas, and hydropic degeneration of the renal tubules was observed in group B. Moreover, marked congestion 
was observed all over the renal tissues of the same group. While group $C$ displayed significantly lesser glomerular and tubular damage as compared with comparison summary of all the histopathological changes in renal tissues observed in each group under light microscopy.

group B. Table III demonstrates the grade-wise

\begin{tabular}{|l|c|c|c|c|}
\hline \multicolumn{1}{|c|}{ Table I: Difference in mean body weight and absolute kidney weight between experimental groups by Post-hoc } \\
Tukey Test \\
\hline Variables & Group A & Group B & Group C & P-value \\
\hline Initial body weight (gm) & $262.7 \pm 3.8$ & $259.3 \pm 2.4$ & $261.1 \pm 3.1$ & 0.38 \\
\hline Final body weight (gm) & $270.2 \pm 6.4^{b, c}$ & $221.9 \pm 4.1^{a, c}$ & $238.1 \pm 2.7^{a, b}$ & $.001^{*}$ \\
\hline Absolute kidney weight (gm) & $1.41 \pm 0.02^{b}$ & $1.48 \pm 0.09^{a, c}$ & $1.39 \pm 0.04^{b}$ & $.001^{*}$ \\
\hline
\end{tabular}

${ }^{*}$ Statistically significant difference between the groups on ANOVA

$a, b, c$ denotes the statistically significant difference between control and treated groups, respectively through post hoc Tukey test $(P<.05)$.

Table II: Difference in mean serum urea, creatinine, GPX, and CRP between experimental groups by Post-hoc Tukey Test

\begin{tabular}{|l|c|c|c|c|}
\hline Mean Serum Levels & Group A & Group B & Group C & P-value \\
\hline Serum Urea (mg/dl) & $22.70 \pm 2.66^{\mathrm{b}, \mathrm{c}}$ & $57.27 \pm 3.65^{\mathrm{a}, \mathrm{c}}$ & $38.10 \pm 0.99^{\mathrm{a}, \mathrm{b}}$ & $.001^{*}$ \\
\hline Serum Creatinine (mg/dl) & $0.45 \pm 0.05^{\mathrm{b}, \mathrm{c}}$ & $0.78 \pm 0.09^{\mathrm{a}, \mathrm{c}}$ & $0.58 \pm 0.16^{\mathrm{a}, \mathrm{b}}$ & $.001^{*}$ \\
\hline Serum GPX (ng/dl) & $1.44 \pm 0.13^{\mathrm{b}, \mathrm{c}}$ & $0.85 \pm 0.11^{\mathrm{a}, \mathrm{c}}$ & $1.54 \pm 0.07^{\mathrm{a}, \mathrm{b}}$ & $.001^{*}$ \\
\hline Serum CRP (mg/dl) & $0.10 \pm 0.11^{\mathrm{b}, \mathrm{c}}$ & $0.81 \pm 0.25^{\mathrm{a}, \mathrm{c}}$ & $0.57 \pm 0.15^{\mathrm{a}, \mathrm{b}}$ & $.001^{*}$ \\
\hline
\end{tabular}

${ }^{*}$ Statistically significant difference between the groups on ANOVA

$\mathrm{a}, \mathrm{b}, \mathrm{c}$ denotes the statistically significant difference between control and treated groups, respectively through post hoc Tukey $(P$-value $<.05)$

GPX-Glutathione Peroxidase; CRP-C-Reactive Protein

\begin{tabular}{|l|c|c|c|c|}
\hline \multicolumn{5}{|c|}{ Table III: Grade-wise comparison of histopathological changes in renal tissue of rats } \\
\hline & Cellular infiltration & Peritubular Fibrotic Areas & Hydropic Degeneration & Vascular congestion \\
\hline Group A & 0 & 0 & 0 & 0 \\
\hline Group B & $* * *$ & $* * *$ & $* * *$ & $* * *$ \\
\hline Group C & $*$ & $* *$ & $* *$ & $*$ \\
\hline
\end{tabular}

Grading score follows: none $(0)$, mild $\left({ }^{*}\right)$, moderate $\left({ }^{* *}\right)$ and severe $\left({ }^{* * *}\right)$
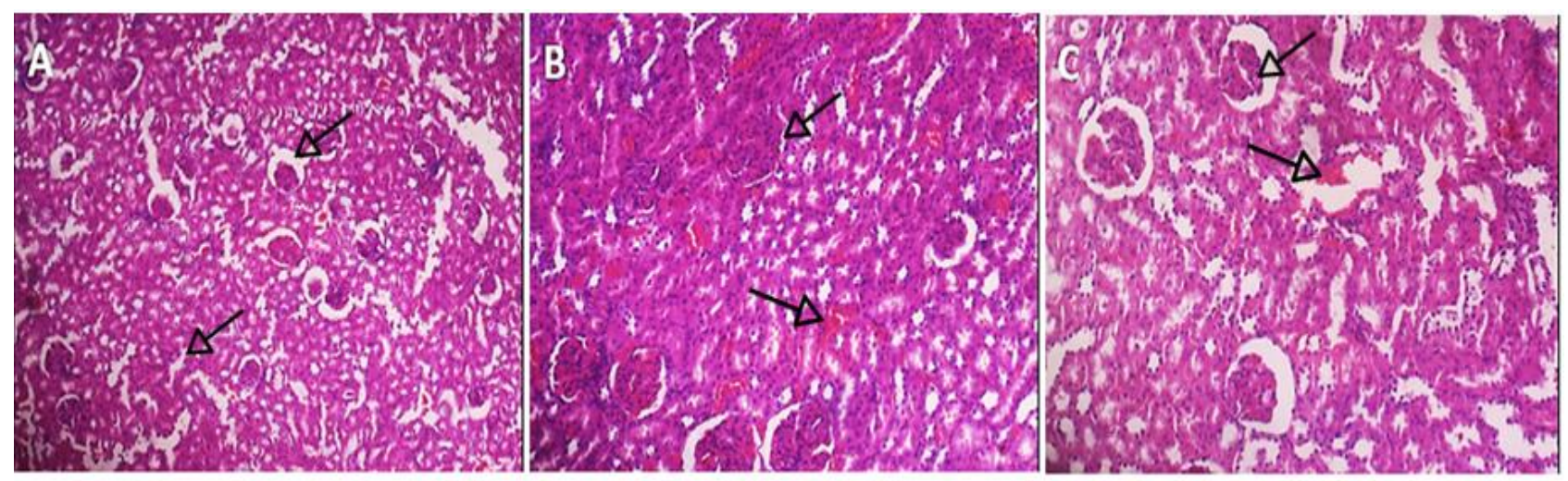

Figure 1. A: Control group shows normal glomerular and tubular architecture (H\&E, 100x). B: Nephrotoxic group shows shrinkage of glomeruli, dilatation of Bowman's slit, and congestion (H\&E, 400x). C: L-arginine group shows near normal glomerular structure and comparatively less apparent renal congestion (H\&E, 400x). 


\section{Discussion}

The present study sought to investigate the nephroprotective effects of L-arginine exerted on cisplatin-induced AKI in a rat model by examining different biochemical and histopathological parameters associated with kidney function of intoxicated and treated rats.

Significant alterations in kidney function and histopathological status were observed in experimental rats intoxicated with cisplatin. However, significant improvement was observed in body weight and all biochemical parameters related to kidney function causing significant reduction in serum creatinine, urea, C-reactive protein, and Glutathione peroxidase levels as well as improving the renal histological architecture. These results show that L-arginine therapy can ameliorate cisplatin-induced nephrotoxicity by improving levels of endogenous antioxidants.

Being the excretory units of the body, the kidneys are exposed to numerous toxic substances causing the liberation of elevated quantities of free radicals which make the kidneys vulnerable to damage. ${ }^{15}$ Cisplatin has been proven to be toxic to kidneys causing alterations in the parameters of renal function. ${ }^{16}$ Cisplatin has also been shown to have a direct inflammatory effect on the kidneys as well as causing increased oxidative stress leading to depletion of vital anti-oxidant molecules like glutathione. ${ }^{17}$ In our experiment, administration of cisplatin was followed by a reduction in the weight of the experimental animals, which is consistent with previous findings. ${ }^{3}$ The animals receiving Larginine therapy showed a much less reduction in their weights, which is consistent with the findings of Eshraghi-Jazi et al., who observed that arginine ameliorates cisplatin-induced weight loss in male rats. $^{18}$

In this study, there was an increase in the blood urea and creatinine of all the animals receiving cisplatin as compared with the controls. These findings are similar to the results reported by Wang et al. and Adeneye and Benebo. ${ }^{19,20}$ However, blood urea and creatinine levels in the rats receiving adjunct therapy of L-arginine were far less than those who received Cisplatin alone which is consistent with the findings of Başhan et al. ${ }^{21}$ Saleh et al. also reported a significant rise in blood urea and creatinine levels in experimental animals which received cisplatin but the experimental animals receiving concomitant arginine therapy showed near normal levels, which is consistent with the current study. ${ }^{22}$

In this experiment, there was an increase in the CRP levels and decrease in the serum GPX levels of cisplatin-treated rats denoting inflammation and oxidative stress, respectively. Such changes were not observed in the control group as animals were not exposed to the nephrotoxic agent. However, arginine therapy prevented the change in the CRP and GPX levels. These findings are in accordance with the findings of $\mathrm{Yu}$ et al., who observed that consumption of arginine rich foods such as nuts was inversely associated with inflammatory biomarkers such as CRP. ${ }^{23}$ Hong et al. also observed arginine consumption was inversely related to CRP levels but directly related to serum GPX levels in rats, which is consistent with the findings of our study. ${ }^{24}$ The positive effects of arginine on oxidative stress have also been studied by Gharibi et al., who reported that L-arginine supplementation increased NO concentration and significantly improved oxidative status. ${ }^{16}$

In our study, histological examination of the kidneys showed clear evidence of cisplatin-induced nephrotoxic injury manifested as marked tubular cell necrosis, vascular congestion, and tubular dilatation, which is consistent with previous findings. ${ }^{3}$ However, these changes were much less evident in the arginine treated animals. These findings are consistent with the findings of Bashan and coworkers, who noted that histopathological 
changes were reversed in the nephrotoxic animals who received L-arginine as well. ${ }^{21}$ The main limitation of our study was lack of funds to assess further parameters of renal function, such as plasma and urinary albumin levels, serum electrolyte levels and urine analysis for further evaluating the renal damage and related complications due to Cisplatin.

\section{Conclusion}

The present study concluded that L-arginine exerts an anti-oxidative, anti-inflammatory, and nephroprotective effect against Cisplatin-induced renal tissue damage. However, further studies should be carried out for a more detailed evaluation of the protective effects of arginine on other organs as well, to give more insight into the topic.

\section{References}

1. Eckardt K-U, Coresh J, Devuyst O, Johnson RJ, Köttgen A, Levey AS, et al. Evolving importance of kidney disease: from subspecialty to global health burden. Lancet. $\quad 2013 ; \quad 382(9887)$ : $158-69$. Doi:10.1016/S0140-6736(13)60439-0.

2. Kianian F, Seifi B, Kadkhodaee $M$, Sajedizadeh A, Ahghari P. Protective effects of celecoxib on ischemia reperfusion-induced acute kidney injury: comparing between male and female rats. Iran J Basic Med Sci. 2019; 22(1): 43-8. Doi: 10.22038/ijbms.2018. 29644.7156.

3. Meghi KA, Talpur RA, Uqaili AA, Nizammani YM, Kazi $\mathrm{N}$, Nizammani GS. Resveratrol attenuates oxidative stress in chemotherapy induced acute kidney injury: an experimental rat model. KMUJ. 2019; 11(2): 85-9. Doi: 10.35845/kmuj.2019.19114.

4. Martinho N, Santos TC, Florindo HF, Silva LC. Cisplatin-membrane interactions and their influence on platinum complexes activity and toxicity. Front Physiol. 2019; 9: 1898. Doi:10.3389/fphys.2018. 01898.

5. Yu W, Chen Y, Dubrulle J, Stossi F, Putluri V, Sreekumar A, et al. Cisplatin generates oxidative stress which is accompanied by rapid shifts in central carbon metabolism. Sci Rep. 2018; 8(1): 1-12. Doi:10.1038/s41598-018-22640-y.

6. Schramm L, Heidbreder E, Lopau K, Schaar J, Zimmermann J, Harlos J, et al. Influence of nitric oxide on renal function in toxic acute renal failure in the rat.
Miner Electrolyte Metab. 1996; 22(1-3): 168-77. PMID: 8676813.

7. Cherla G, Jaimes EA. Role of L-arginine in the pathogenesis and treatment of renal disease. J Nutr. 2004; 134(10 Suppl): 2801S-6S; discussion 18S-19S. Doi: $10.1093 / \mathrm{jn} / 134.10 .2801 S$.

8. Wink DA, Miranda KM, Espey MG, Pluta RM, Hewett SJ, Colton C, et al. Mechanisms of the anti-oxidant effects of nitric oxide. Antioxid Redox Signal. 2001; 3(2): 203-13. Doi:10.1089/152308601300185179.

9. Omi $H$, Okayama $N$, Shimizu $M$, Fukutomi $T$, Nakamura A, Imaeda K, et al. Cilostazol inhibits high glucose-mediated endothelial-neutrophil adhesion by decreasing adhesion molecule expression via NO production. Microvasc Res. 2004; 68(2): 119-25. Doi: 10.1016/j.mvr.2004.05.002.

10. Charan J, Biswas T. How to calculate sample size for different study designs in medical research? Indian J Psychol Med. 2013; 35(2): 121-26. Doi: 10.4103/0253-7176.116232.

11. Hanif MS, Baloch MS, Meghji KA, Abbas A, Kashif S, Qureshi R. Histopathological changes in the gastric mucosa induced by carbaryl toxicity: an experimental rat model. KMUJ. 2020; 12(2): 137-42. Doi:10.35845/kmuj.2020.19896.

12. Claybaugh T, Decker S, McCall K, Slyvka Y, Steimle J, Wood $A$, et al. L-arginine supplementation in type II diabetic rats preserves renal function and improves insulin sensitivity by altering the nitric oxide pathway. Int J Endocrinol. 2014; 2014: 171546. Doi:10.1155/ 2014/17154.6

13. Sahu BD, Kumar JM, Sistla R. Baicalein, a bioflavonoid, prevents cisplatin-induced acute kidney injury by upregulating antioxidant defenses and down-regulating the MAPKs and NF-KB pathways. PloS one. 2015; 10(7): e0134139. Doi:10.1371/journal.pone.0134139

14. Bazmandegan G, Amirteimoury $M$, Kaeidi $A$, Shamsizadeh A, Khademalhosseini $M$, Nematollahi $\mathrm{MH}$, et al. Sumatriptan ameliorates renal injury induced by cisplatin in mice. Iran J Basic Med Sci. 2019; 22(5): 563-67. Doi:10.22038/ijbms.2019 .33620 .8020 .

15. Osman A-MM, Telity SA, Damanhouri ZA, Al-Harthy SE, Al-Kreathy HM, Ramadan WS, et al. Chemosensitizing and nephroprotective effect of resveratrol in cisplatin-treated animals. Cancer Cell Int. 2015; 15: 6. Doi:10.1186/s12935-014-0152-2.

16. Gharibi F, Soltani N, Maleki M, Talebi A, Nasiri M, Shirdavani $S$, et al. The Protective Effect of L-arginine in Cisplatin-induced Nephrotoxicity in Streptozotocin-induced Diabetic Rats. Adv Biomed Res. 2017; 6: 100. Doi:10.4103/2277-9175.212928. 
17. Manohar S, Leung N. Cisplatin nephrotoxicity: a review of the literature. J Nephrol. 2018; 31(1): 1525. Doi:10.1007/s40620-017-0392-z.

18. Eshraghi-Jazi F, Nematbakhsh $M$, Nasri $H$, Talebi $A$, Haghighi M, Pezeshki Z, et al. The protective role of endogenous nitric oxide donor (L-arginine) in cisplatin-induced nephrotoxicity: Gender related differences in rat model. J Res Med Sci. 2011; 16(11): 1389. PMID:22973338.

19. Wang H, Jia Z, Sun J, Xu L, Zhao B, Yu K, et al. Nitrooleic acid protects against cisplatin nephropathy: role of COX-2 /mPGES-1 /PGE2 cascade. Mediators Inflamm. 2015; 2015: 293474. Doi:10.1155/2015/293474.

20. Adeneye AA, Benebo AS. Chemopreventive Effect of Tadalafil in Cisplatin-Induced Nephrotoxicity in Rats. Niger J Physiol Sci. 2016; 31(1): 1-10.

21. Bashan I, Bashan P, Secilmis MA, Singirik E. Protective effect of L-arginine on gentamicin-induced nephrotoxicity in rats. Indian J Pharmacol. 2014; 46(6): 608-12. Doi:10.4103/0253-7613.144915.

22. Saleh S, El-Demerdash E. Protective effects of Larginine against cisplatin-induced renal oxidative stress and toxicity: role of nitric oxide. Basic Clin Pharmacol Toxicol. 2005; 97(2): 91-7. Doi: 10.1111/j.1742-7843.2005.pto_114.x.

23. Yu Z, Malik VS, Keum N, Hu FB, Giovannucci EL, Stampfer MJ, et al. Associations between nut consumption and inflammatory biomarkers. Am J Clin Nutr. 2016; 104(3): 722-8. Doi:10.3945/ajen.116.134205.

24. Hong MY, Beidler J, Hooshmand S, Figueroa A, Kern $M$. Watermelon and L-arginine consumption improve serum lipid profile and reduce inflammation and oxidative stress by altering gene expression in rats fed an atherogenic diet. Nutr Res. 2018; 58: 46-54. Doi: 10.1016/j.nutres.2018.06.008. 